epl draft

\title{
Boundary layer (shear-band) in frustrated viscoplastic flows
}

\author{
T. CHEVALIER, S. RODTS, X. CHATEAU, J. BOUJLEL, M. MAILLARD, P. COUSSOT \\ Université Paris-Est, Laboratoire Navier, 2 Allée Kepler, 77420 Champs sur Marne, France
}

PACS 83.60. La - Viscoplasticity

PACS $47.20 . \mathrm{GV}$ - Viscous and viscoelastic instabilities

PACS $62.20 . \mathrm{F}--$ Deformation and plasticity

\begin{abstract}
We show that frustrated creep flows of yield stress fluids give rise to a boundary layer, which takes the form of a liquid region of uniform significant thickness separating two solid regions. In this boundary layer the shear rate is approximately constant for a given flow rate and the layer thickness extremely slowly varies with the flow rate.
\end{abstract}

Yield stress fluids such as concentrated colloids, emulsions, foams, are jammed systems which behave as liquids if they are submitted to a sufficiently large stress, and as solids otherwise [1-2]. In usual situations the stress is heterogeneous throughout the material so that "solid" regions coexist with "liquid" regions. In simple uniform flows (e.g. in straight conduits or open channels, in Couette geometry, etc) the solid-liquid interface reaches the boundaries when the velocity tends to zero, so that the liquid region tends to disappear for creep flows [3-4]. A similar result is obtained for thixotropic yield stress fluids which develop shear-banding in channelized or concentric cylinder flows [5-6]. Another type of shear-banding was also observed in starting (Couette) flow of simple yield stress fluids [7]. The recent generalized theoretical approach of shearbanding [8] for time-dependent flows also considered uniform flows. On the contrary, in pure solids although shear-banding can also occur in simple shear [9] it is currently observed in more complex deformation fields [10].

A wide range of practical operations in civil engineering (paints, mortars, concrete, drilling fluids) in food industry and cooking (purees, sauces, dough), or in cosmetic applications (gels, foams, creams), involve flows of yield stress fluids initially at rest and partially removed by some flow or by the displacement of an object through it: mixing, injection, formwork filling, coating on walls or skins, etc. Such a problematic is typical of yield stress fluids and to what extent the fluid initially in its solid regime is liquefied and/or removed is a crucial question to address in order to minimize the required energy and improve the efficiency of the process. However our knowledge of these flows is extremely poor and essentially concerns the displacement of solid spheres or cylinders through yield stress fluids [11]. Even in that case the complexity of the problem and the difficulty to measure internal flow characteristics in usual pasty materials precluded a full understanding. For example it was shown that in some cases the elasticity of the material in its solid regime can allow the sphere to displace through it by liquefying only an extremely small volume of material [12].

As a consequence one needs to have a detailed information on these frustrated viscoplastic flows, in which due to the geometrical conditions and the yielding character of the fluid there necessarily exists a region which will not flow. Here we focus on two typical simple situations of that type, i.e. (i) the injection of a yield stress fluid through a box initially fulfilled with the same fluid and (ii) the displacement of a long object through a yield stress fluid lying in a container. We show that in both cases the motion relies on the existence of a boundary layer, which takes the form of a liquid region of uniform significant thickness separating two solid regions. Surprisingly, in this boundary layer the shear rate is approximately constant and the layer thickness extremely slowly varies with the flow rate.

Our first experiment consists in the injection of a yield stress fluid in a cylindrical box (diameter and length: $7 \mathrm{~cm}$ ) from a coaxial smaller conduit of radius $R=17.5 \mathrm{~mm}$. The fluid is a concentrated inverse emulsion exhibiting a yield stress $\left(\tau_{c}\right)$ of $74 \mathrm{~Pa}$, and with a simple shear flow curve well represented by a HerschelBulkley (HB) model $\left(\tau=\tau_{c}+k \dot{\gamma}^{n}\right.$, in which $\tau$ is the shear stress and $\dot{\gamma}$ the shear rate) with $k=13.5 \mathrm{~Pa}_{\mathrm{s}} \mathrm{s}^{n}$ and $n=0.4$. The material was prepared by dispersing a water $+\mathrm{CaCl} 2(150 \mathrm{~g} / \mathrm{l})$ salt solution in a surfactant solution (dodecane (Acros organics) $+7.5 \%$ wt of Span 80 surfactant) in a Silverson mixer. For the tests the material is supplied via an extrusion syringe $(8 \mathrm{~cm}$ diameter, $1 \mathrm{~m}$ long) to the small conduit in which a uniform flow is established at a significant distance from the entrance 
in the box [4]. The flow rate is imposed by a piston in the syringe, leading to an average velocity $(V)$ in the small conduit ranging from 0.16 to $9.4 \mathrm{~mm} / \mathrm{s}$. For these flows the generalized Reynolds number (i.e. $\operatorname{Re}=\rho V^{2} / \tau_{c}$, in which $\rho$ is the fluid density) is smaller than $10^{-3}$. This means that we are dealing with creep flows solely governed by viscous effects. Under these conditions the flow characteristics depend on the boundary conditions and the value of the Bingham number, which estimates the ratio of the constant (yielding) to the rate-dependent (viscous) parts of the constitutive equation: $B i=\tau_{c} R^{n} / k V^{n}$, and finally expresses the relative importance of solid to liquid effects. Note that in this expression we arbitrarily used the apparent shear rate $V / R$ in the small conduit.

The extrusion syringe is run by a home made mechanical press made of materials with low electric conductivity and magnetic susceptibility so as to ensure compliance with MRI electromagnetic fields. The whole set-up is inserted vertically in the channel of a vertical proton MRI system (Avance 24/80 DBX by Bruker, 0.5T superconducting magnet by Oxford, $20 \mathrm{~cm}$ inner diameter). Measurements are performed directly on hydrogen nuclei of the fluid itself, so that no additional tracer particles are necessary. "Velocity maps" were recorded in a $1 \mathrm{~cm}$ thick vertical slice including the central axis with the help a home made MRI procedure based on standard concepts of the 'spin warp imaging - phase encoded velocity' [13]. Since inertia effects were negligible the flow was symmetrical by rotation around the central axis and we observed only in-plane components. Despite the low velocities studied here we could obtain a sufficiently large signal to noise ratio thanks to recent methodological improvements [4, 6, 14]. The voxel size in radial and axial directions in the Fourier space was 1 and $4 \mathrm{~mm}$ respectively and the absolute uncertainty on velocity values for a given experiment was $3 \%$ of the maximum velocity in the small conduit.

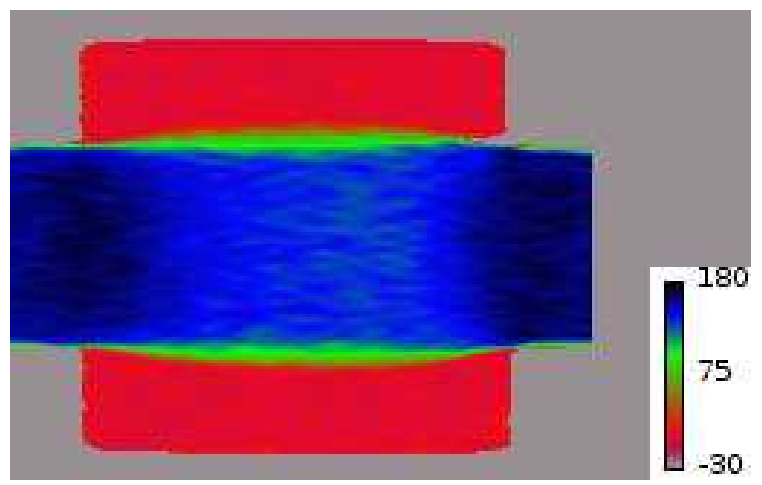

Fig. 1: Velocity map in a longitudinal cross-section for $V=0.16 \mathrm{~mm} / \mathrm{s}$. The velocity amplitude (in microns per second) in each voxel is represented by a color according to the scale at the bottom right.
A typical velocity field is shown in Figure 1. The flow is composed of three main parts: a region (blue in Figure 1) around the central axis in which the velocity is approximately uniform, a layer (green) around this region in which the velocity decreases radially, and an outer region (red) along the box walls where the fluid is apparently at rest.

The flow characteristics in the small duct (see Figure 2) just before the entrance and after the exit from the box are those expected for the steady uniform flow in a straight conduit: a central plug surrounded by a liquid layer in contact with the wall [2-3]. Indeed, under such conditions we have a simple shear along the axis direction (the non-zero component of the velocity, i.e. $v$, only depends on $r$, the distance from the central axis) and the momentum equation along the conduit axis ( $z$, with $z=0$ at the entrance in the box) writes: $-\nabla p+\partial(r \tau) / r \partial r=0$, where $\nabla p=\partial p / \partial z$ is the pressure gradient. Assuming a homogeneous pressure in each cross-section $(p=p(z))$ we deduce that the shear stress expresses as $\tau=(r / 2) \nabla p$. Note that $\tau$ is negative because the fluid advances faster for smaller $r$, and the pressure drop, $\nabla p$, is negative. Since the material remains in its solid regime as long as $|\tau|<\tau_{c}$ we deduce that the fluid flows as a plug (solid region) inside the region $r<r_{c}=-2 \tau_{c} / \nabla p$. Using the $\mathrm{HB}$ constitutive equation and the above stress distribution the velocity is deduced by integration: $r \leq r_{c} \Rightarrow v_{0}=\alpha\left(r_{0} / r_{c}-1\right)^{m+1}$; $r>r_{c} \Rightarrow v(r)=v_{0}-\alpha\left(r / r_{c}-1\right)^{m+1}$, in which $m=1 / n$ and $\alpha=(1 / 1+m) r_{c}\left(\tau_{c} / k\right)^{m}$. Using the rheological parameters measured independently (see above) this theory appears to predict very well the velocity profile observed in the small conduit even just before the entrance (see Figure 2).

Another integration of the velocity distribution provides the relation between the pressure drop and the average velocity $(V)$ :

$$
\begin{aligned}
& B i^{-m}=G^{-3}(G-1)^{m+1} \\
& \quad \times\left[\left((m+1)(m+2) G^{2}+2(m+1) G+2\right) /(m+1)(m+2)(m+3)\right]
\end{aligned}
$$

in which $G=-R \nabla p / 2 \tau_{c}$. For $B i>>1$ this equation reduces to: $G \approx 1+(m+1)^{1 /(m+1)} B i^{-m / 1+m}$, so that the thickness of the liquid region (along the wall), i.e. $e=R-r_{c}=-\left(2 \tau_{c} / \nabla p\right)(G-1)$, writes:

$$
e / R \approx(m+1)^{1 /(m+1)} B i^{-m / 1+m}
$$

Within the box the flow characteristics are quite different from those of a uniform flow: the moving fluid is surrounded by a wide region of fluid at rest (see Figure 
1). This contrasts with what is observed with simpler fluids: Newtonian or viscoelastic fluids flow at any point, possibly with some regions of reverse vorticity in the corners [15]. For yield stress fluids such a situation is approached for $B i<<1$, i.e. i.e. when the impact of the yield stress character becomes minor: in that case it was shown [16] that the shape of the liquid region becomes significantly rounded and occupies a wider region of the box when $B i$ decreases.

In our case, except around the corners just after the entrance and just before the exit of the box the radial component of the velocity is negligible. Finally, despite the complexity of the boundary conditions, after a slight radial expansion the moving region includes a region of uniform flow: the velocity profiles between 2 and $5 \mathrm{~cm}$ from the entrance are apparently identical (see Figure 2 ). The velocity profile is composed of a plug region (central plateau) and an outer sheared region. Surprisingly the slope of the velocity profile in the latter region is approximately constant (see Figure 2) so that the shear rate in this region of thickness $\delta$ is almost uniform: it is roughly equal to $V / \delta$. This result differs from the uniform flow through a solid conduit for which the shear rate strongly varies from zero along the plug to its maximum value at the wall (see Figure 2).

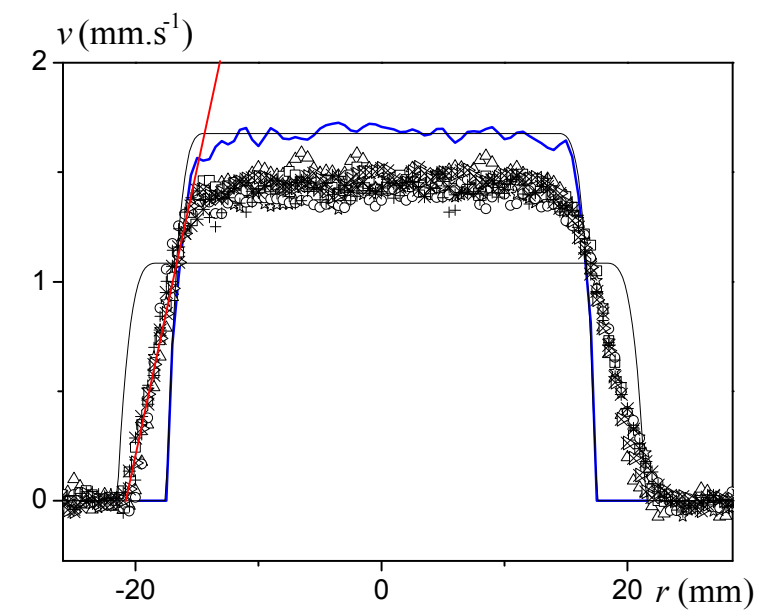

Fig. 2: Flow of a yield stress fluid through a box $(V=1.6 \mathrm{~mm} / \mathrm{s})$ : longitudinal velocity as a function of the distance from the axis at different distances from the entrance: $-0.5 \mathrm{~cm}$ (blue line); then every $0.5 \mathrm{~cm}$ from $z=2 \mathrm{~cm}$ to $z=5 \mathrm{~cm}$ (symbols). The upper black line corresponds to the simple theory for a uniform flow for a conduit radius $17.5 \mathrm{~mm}(R)$ (i.e. small duct). The lower black line corresponds to the same theory for a conduit of radius $21.5 \mathrm{~mm}\left(r_{0}\right)$. The inclined red straight line is a guide for the eye.
Finally, in the central region inside the box we have a steady flow of yield stress material through a straight cylindrical solid region of radius $r_{0} \approx 21.5 \mathrm{~cm}$ composed of yield stress fluid at rest, but the velocity profile predicted by the above theory in that case strongly differs from the measured velocity profile (see Figure 2). Actually, inside the box the stress distribution obtained for the uniform flow is not compatible with the material behavior in the outer solid regions. Indeed if $\tau=(r / 2) \nabla p$ we have $\left|\tau\left(r_{0}\right)\right|>\tau_{c}$ whereas the basic conditions for the fluid to remain in its solid state in the outer region within the box is $\left|\tau\left(r_{0}\right)\right| \leq \tau_{c}$. Moreover, a velocity profile such as that observed in Figure (2) with an almost constant slope in the sheared region implies that the shear stress is approximately constant over some significant distance. Thus, for increasing radial distance $\tau$ first increases from $\tau_{c}$ then reaches a kind of plateau and finally decreases towards $\tau_{c}$. From the momentum equation we deduce that the pressure gradient is negative close to the plug but positive along the outer, stopped, fluid region inside the box. This suggests that in some cases a similar complex pressure gradient field might take place during the start up flow of a yield stress fluid leading to a transient shear-banding [7].

Such a situation results from the frustrated character of the present flow, which implies the existence of a significant volume of arrested fluid. If the box was replaced by a much longer box, as usual a standard uniform flow would be recovered beyond some distance from the entrance, with now a liquid region restricted to a thin layer along the box wall with a thickness described by (1).

Let us now look more precisely at the velocity profiles obtained in the uniform region for different flow rates. At first sight they are close to each other when the velocity is rescaled by the average velocity (see Figure 3 ). This means that the thickness of the central plug and the thickness of the sheared region are almost independent of the velocity. A more precise look at the data shows that the sheared thickness $\delta$ increases from 5 to 8.2 $\mathrm{mm}( \pm 10 \%)$ when $V$ increases from 0.016 to $9.4 \mathrm{~mm} / \mathrm{s}$ (see inset of Figure 3). For the uniform flow through a straight duct of similar radius, according to (1) $e$ should vary much more widely, i.e. between 1.8 and 6.2 $\mathrm{mm}$, which again illustrates the difference between the flow characteristics inside the box and those for a uniform flow. 


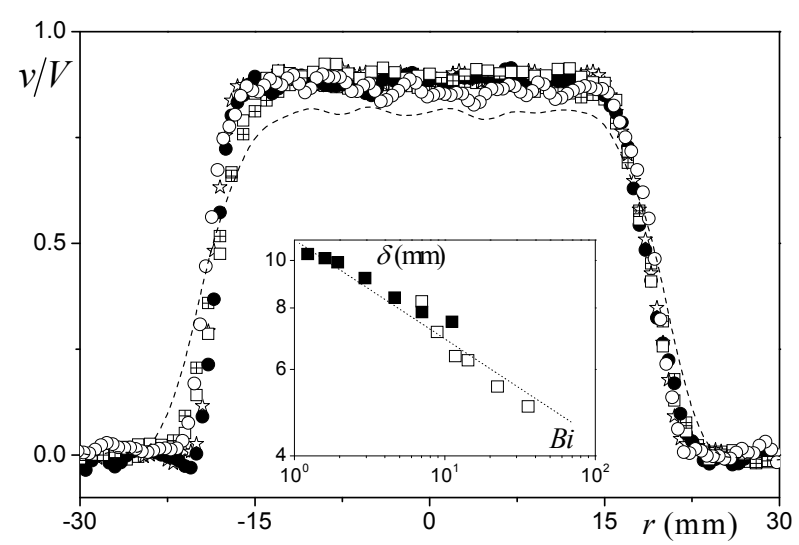

Fig. 3: Profile of the longitudinal velocity (scaled by the average velocity in the small conduit) for the flow of yield stress fluid through a box for $V=0.16 \mathrm{~mm} / \mathrm{s}$ (open circles), $0.52 \mathrm{~mm} / \mathrm{s}$ (stars), $1.6 \mathrm{~mm} / \mathrm{s}$ (filled circles), $2.6 \mathrm{~mm} / \mathrm{s}$ (open squares), $5.2 \mathrm{~mm} / \mathrm{s}$ (cross squares), and $9.4 \mathrm{~mm} / \mathrm{s}$ (dashed line). Inset: Boundary layer thickness for flow through a box (open squares) and plate penetration (filled squares) as deduced from above data and those of Figure 4, as a function of the Bingham number; the dotted line has a slope $-1 / 5$ in logarithmic scale.

The almost constant shear rate observed in the liquid region suggests that some shear-banding effect occurs. However this phenomenon differs from usual shearbanding in particular observed with micelles [17] or thixotropic suspensions [5-6]. Indeed here there is no intrinsic critical shear rate below which one starts to observe shear-banding. The apparent shear rate in the sheared band $(V / \delta)$ is simply roughly proportional to $V$.

It is also remarkable that a wide central plug region also exists within the flow inside the box, with a size close to that of the plug in the smaller conduit. According to the technique developed in [18] we computed the total deformation undergone by the fluid elements along their trajectories. We found that except in an outer layer of a few millimetres the fluid which was in the upstream plug region undergoes a total deformation smaller than 0.1 until reaching the central plug region. Since in simple shear the critical deformation beyond which the emulsion used turns from a solid to a liquid is about 0.2 this means that in our flow most of the central fluid part remains in its solid regime during its motion through the box. The whole flow may thus be seen as a simple erosion of the plug while it flows through the box, with an eroded thickness almost uniform and almost independent of the flow rate in our range of $B i$.

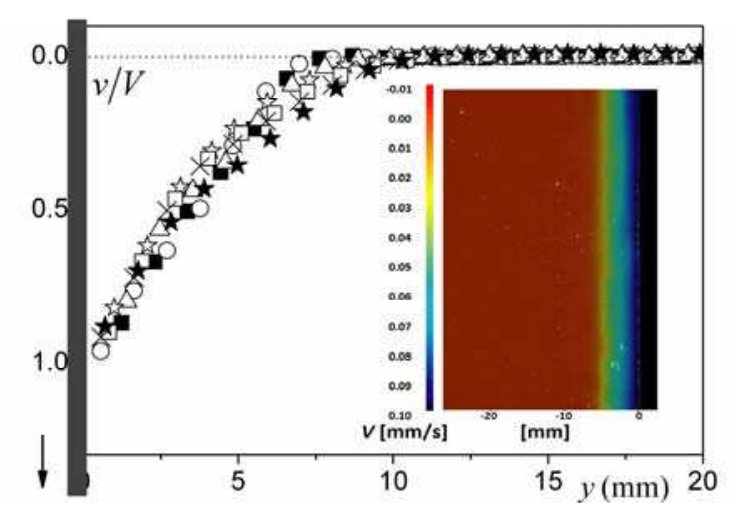

Fig. 4: Plate (1.8 mm thick) moving vertically through a bath of yield stress fluid (well described by a HB model with

$\tau_{c}=59.3 \mathrm{~Pa}, k=23.6 \mathrm{~Pa} \cdot \mathrm{s}^{n}$ and $n=0.38$ ) (data from [10]): velocity profiles (scaled by the plate velocity) measured by PIV at a distance of $15 \mathrm{~cm}$ from the plate tip as a function of the distance from the plate for different plate velocities: 0.03 (filled squares), 0.1 (open circles), 0.3 (triangles), 1 (stars), 3 (open squares), 5 (crosses), $10 \mathrm{~mm} / \mathrm{s}$ (stars). Inset: view of the velocity field along the plate (black region) between 10 and $15 \mathrm{~cm}$ from the plate tip for $0.1 \mathrm{~mm} / \mathrm{s}$ plate velocity. The straight line is a guide for the eye.

Let us now turn to another simple frustrated flow: the displacement of a solid object through a yield stress fluid macroscopically at rest in a container. We recently carried out such experiments by moving vertically a plate through a Carbopol solution lying in a wide container [18]. We can re-analyze these data in the light of our results of the flow through a box. Beyond a distance, from the plate tip penetrating the fluid, of about 10 times the plate thickness, there is along the plate a liquid region of uniform thickness (see inset of Figure 4) while the rest of the fluid is deformed in its solid regime. This situation strongly differs from the boundary layer in a simple viscous liquid at low Reynolds number which extends to a distance increasing with the distance from the plate tip [19]. Moreover, here the tangential velocity profiles (see Figure 4) in this uniform liquid region exhibit two trends quite similar to those found for the flow through the box: (i) at first sight they are similar when rescaled by the plate velocity; (ii) for each curve the slope varies in a limited range so that as a first approximate the shear rate is constant. We thus have a similar boundary layer effect in both flow types.

Let us consider the theory for such flow type. For a uniform flow along the plate in a layer of thickness $\delta$ the only non-zero component of the velocity $v$ is parallel to the plate and only depends on the distance from the plate $y$. The momentum equation then writes: 
$\partial p / \partial x=d \tau / d y ; \partial p / \partial y=0$, from which we deduce $\tau(y)=\tau_{w}-\left(\tau_{w}-\tau_{c}\right) y / \delta$ for $0<y<\delta$, where $\tau_{w}=\tau(y=0)$ is the shear stress at the wall and $\delta=-\left(\tau_{w}-\tau_{c}\right) /(\partial p / \partial x)$. Equating this stress with that given by the HB constitutive equation of the material in the liquid regime we find an expression for the shear rate $\dot{\gamma}=d u / d y$ which may be integrated to give the velocity profile: $v=V(1-y / \delta)^{1+1 / n}$ for $0<y<\delta$. The shape of this theoretical velocity profile is in good agreement with the experimental ones (see [18]). However in this theory $\delta$ is a fitting parameter related to the pressure gradient, $\partial p / \partial x$, but not necessarily independent of the velocity.

In order to solve this problem Piau [20] assumed that the stress distribution in the solid region can be found from the solution for a plate of finite length $2 a$ immersed in an elastic medium and imposing to the fluid a shear stress equal to the yield stress along its main direction. He found that the pressure gradient may be approximated by a constant value: $\nabla p=-2 \tau_{c} / \pi a$. In the limit of large Bingham numbers an explicit expression for the boundary layer thickness is then obtained [21]:

$$
\delta / a \propto B i^{-m / m+1}
$$

We can see that the variations of the thickness as a function of the velocity as predicted by this theory, which is similar to that found for the uniform flow through a conduit (equation (1), is much faster than the variations observed in our experiments.

For the flow through the box the thickness of the boundary layer should depend on the box length and the upper conduit radius. For the plate penetration it was found that the stress along the plate in the region of uniform flow was independent of the plate thickness over a range of about one decade [22]. This stress is directly deduced from the slope of the velocity profile along the wall, a slope which governs the shape of this profile and in particular its extent. This suggests that in some significant range the sheared thickness is also poorly dependent of the geometrical conditions. Its exact dependence on the geometrical characteristics still constitutes a wide field of research but it remains that for given geometrical conditions $\delta$ is expected to vary only as a function of $B i$. Here our critical observation for these two frustrated flows is that $\delta$ very slowly increases when $B i$ increases.

It appears that the variations of $\delta$ as a function of $B i$ are similar for these two frustrated flows (see inset of Figure 3), i.e. we have $\delta \propto B i^{-1 / \alpha}$ with $\alpha=5 \pm 0.4$, which implies $\delta \propto V^{1 / \beta}$ with $\beta=13 \pm 1$ for our $n$ values. The fact that the absolute levels for $\delta(B i)$ are consistent, approximately leading to a single curve, is likely fortuitous since we are dealing with quite different situations and the lengthscales used in $B i$ were chosen arbitrarily (conduit radius and plate thickness). Nevertheless the similarity of these values tends to suggest some global consistency of this boundary layer effect for frustrated flows.

Finally our results show that frustrated creep flows of viscoplastic materials along a sufficiently long distance tend to develop in the form of the relative motion of two solid regions separated by a liquid region, i.e. a boundary layer, in which the shear rate is approximately constant, like in a shear-band. The critical result is that the thickness of this boundary layer extremely slowly decreases for increasing Bingham number so that it will appear as constant in most practical situations. This would suggest an original explanation to the shearbanding observed in flows of plastic materials [23], i.e. soils, metals, granular materials, such as in triaxial tests or in the Casagrande shear box which may be considered as typically frustrated flows. In that case the boundary layer thickness is significantly smaller than the values found here for usual yield stress fluids, but this might be consistent with a much larger Bingham number due to a likely much higher $\tau_{c} / k$ value for such materials if described as yield stress fluids.

This boundary layer effect plays a critical role in various practical cases as it implies that even for a flow with complex boundary conditions solid regions can still occupy most of the fluid volume while the interactions between them is limited to a relatively thin liquid layer. For simple yield stress fluids this boundary layer tends to maintain, along the interface with a solid object, a liquid flow in a layer of significant thickness. On the contrary, for thixotropic yield stress fluids we can anticipate that this effect leads to a much stronger localization due to the probable viscosity drop in the sheared region, as effectively observed along the dead zones in extrusion flows [14].

\section{REFERENCES}

[1] A.J. Liu, and S.R. Nagel, Nature, 396, 21 (1998)

[2] P. Coussot, Rheometry of pastes, suspensions and granular materials (Wiley, New York, 2005)

[3] R.B. Bird, D. Gance, B.J. Yarusso, Rev. Chemical Eng., 1, 1 (1982)

[4] B.D. Rabideau, P. Moucheront, F. Bertrand, S. Rodts, N. Roussel, C. Lanos, P. Coussot, J. Non-Newt. Fluid Mech., 165, 394 (2010)

[5] P.C.F. Moller et al., Phys. Rev. E, 77, 041507(2008)

[6] F. Pignon, A., Magnin, and J.M. Piau, J. Rheol. 40, 573-587 (1996); P. Coussot, et al., Phys. Rev. Lett., 88, 175501 (2002); G. Ovarlez et al., Rheol. Acta, 48, 831 
T. Chevalier et al.

(2009); B. Derakhshandeh, D. Vlassopoulos, S.G. Hatzikiriakos, Rheol. Acta, 51, 201 (2012)

[7] T. Divoux, C. Barentin, S. Manneville, Soft Matter, 7, 8409 (2011); T. Divoux et al., Soft Matter, 8, 4151 (2012)

[8] R.L. Moorcroft, and S.M. Fielding, to appear in Phys. Rev. Lett., arXiv:1201.6259 [cond-mat.soft]

[9] L. Anand, K.H. Kim, T.G. Shawki, J. Mech. Phys. Solids, 35, 407 (1987)

[10] A. Nadai, Theory of flow and fracture of solids (McGraw-Hill, New York, 1950)

[11] A.N. Beris et al., J. Fluid Mech., 158, 219 (1985); D.D. Atapattu, R.P. Chhabra, and P.H.T. Uhlherr, J. Non-Newtonian Fluid Mech., 59, 245 (1995); D. Tokpavi, L. Dodji, A. Magnin, P. Jay, J. Non-Newt. Fluid Mech., 154, 65 (2008); A. Putz, I.A. Frigaard, J. NonNewtonian Fluid Mech., 165, 263 (2010)

[12] H. Tabuteau, F. Oppong, J. de Bruyn, P. Coussot, Europhys. Letters , 78, 68007 (2007)

[13] S. Stapf and S. Han, NMR Imaging in Chemical Engineering (Wiley, New York, 2006); P.T. Callaghan, Rep. Progr. Phys., 62, 599 (1999)

[14] S. Rodts et al., Physical Review E, 81, 021402 (2010)

[15] D.J. Tritton, Physical Fluid Dynamics (Oxford Science Publication, Oxford, 1988); D.V. Boger and K. Walters, Rheological Phenomena in Focus, Rheology Series, 4 (Elsevier, Amsterdam, 1993)

[16] P.R. de Souza Mendes, M.F. Naccache, P. R. Varges, F.H. Marchesini, J. Non-Newt. Fluid Mech., 142, 207-217 (2007)

[17] H. Rehage, and H. Hoffmann, Mol. Phys., 74, 933 (1991); D. Roux, F. Nallet, and O. Diat, Europhys. Lett., 24, 53 (1993); E. Cappelaere, J.F. Berret, J.P. Decruppe, R. Cressely, and P. Lindner, Phys. Rev. E, 56, 1869 (1997)

[18] J. Boujlel et al., J. Rheol., 56, 1083 (2012)

[19] R.B. Bird, W.E. Stewart, E.N. Lightfoot, Transport Phenomena (Wiley, New York, 2007)

[20] Piau, J.M., Viscoplastic boundary layer, J. NonNewt. Fluid Mech., 102, 193-218 (2002)

[21] Piau, J.M., K. Debiane, The adhesive or slippery flat plate viscoplastic boundary layer for a shearthinning power-law viscosity, J. Non-Newt. Fluid Mech., 117, 97-107 (2004)

[22] J. Boujlel, P. Coussot, Rheol. Acta, 51, 867 (2012)

[23] J.R. Rice, In Koiter, W.T., ed., Theoretical and Applied Mechanics 207-220 (Amsterdam, NorthHolland, 1977) 